1

2

\title{
Dietary salt promotes cognitive impairment through tau phosphorylation
}

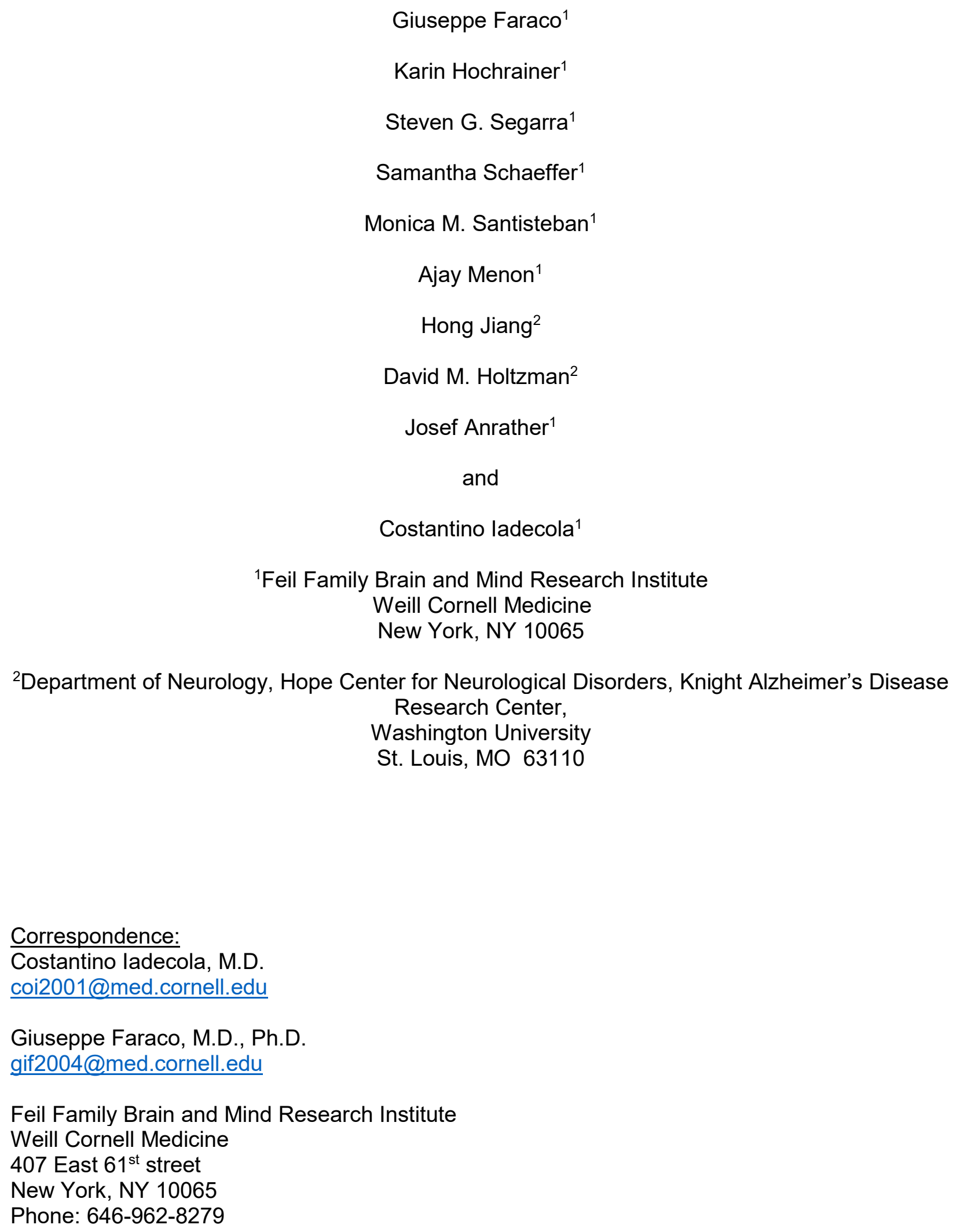


Faraco et al. / page 2

1 Dietary habits and vascular risk factors promote both Alzheimer's disease and cognitive

2 impairment caused by vascular factors ${ }^{1-3}$. Furthermore, accumulation of

3 hyperphosphorylated tau, a microtubule associated protein and a hallmark of Alzheimer's

4 pathology, is also linked to vascular cognitive impairment ${ }^{4-7}$. In mice, a salt-rich diet leads

5 to cognitive dysfunction associated with a nitric oxide deficit in cerebral endothelial cells

6 and cerebral hypoperfusion ${ }^{8}$. Here we report that dietary salt induces tau

7 hyperphosphorylation followed by cognitive dysfunction, effects prevented by restoring

8 endothelial nitric oxide production. The nitric oxide deficiency reduces neuronal calpain

9 nitrosylation resulting in enzyme activation, which, in turn, leads to tau phosphorylation

10 by activating cyclin dependent kinase-5. Salt-induced cognitive impairment is not

11 observed in tau-null mice or in mice treated with anti-tau antibodies, despite persistent

12 cerebral hypoperfusion and neurovascular dysfunction. These findings unveil a causal link

13 between dietary salt, endothelial dysfunction and tau pathology, independent of

14 hemodynamic insufficiency. Avoiding excessive salt intake and maintaining vascular

15 health may help stave off vascular and neurodegenerative pathologies underlying late-life

16 dementia.

Vascular risk factors including excessive salt consumption have long been associated with cerebrovascular dysfunction and cognitive impairment ${ }^{1-3}$. A diet rich in salt promotes stroke and

20 dementia independently of hypertension ${ }^{9-12}$ and has been linked to the cerebral small vessel

21 disease underlying vascular cognitive impairment ${ }^{13,14}$, a condition associated with endothelial

22 dysfunction and reduced cerebral blood $(\mathrm{CBF})^{15}$.

23 Accumulation of the microtubule associated protein tau is a pathological hallmark of

24 Alzheimer's disease ${ }^{16}$. Excessive tau phosphorylation promotes the formation of insoluble tau

25 species, thought to mediate neuronal dysfunction and cognitive impairment ${ }^{17,18}$. Tau

26 hyperphosphorylation and aggregation have increasingly been detected also in vascular brain 
Faraco et al. / page 3

1 pathologies both in humans and animal models ${ }^{4-6}$, and have been linked to cognitive dysfunction

2 in patients with small vessel disease ${ }^{7}$.

3 In mice, a high salt diet (HSD) induces cognitive dysfunction by targeting the cerebral

4 microvasculature through a gut-initiated adaptive immune response mediated by Th17

5 lymphocytes ${ }^{8}$. The resulting increase in circulating IL17 leads to inhibition of endothelial nitric

6 oxide (NO) synthase (eNOS) and reduced NO production in cerebral microvessels, which, in turn,

7 impairs the endothelial regulation of microvascular flow and lowers cerebral blood flow (CBF) by

$8 \approx 25 \%{ }^{8}$. Remarkably, the increases in CBF evoked by neural activity and blood-brain permeability

9 are not altered ${ }^{8}$. However, it remains unclear how hypoperfusion, in HSD as in other vascular risk

10 factors, leads to impaired cognition. The prevailing view is that reduced CBF compromises the

11 delivery of oxygen and glucose to energy-demanding brain regions involved in cognitive

12 function ${ }^{15,19}$. But the relatively-small reductions in CBF associated with $\mathrm{HSD}^{8}$ and vascular

13 cognitive impairment ${ }^{20}$ do not reach the threshold needed to induce sustained cognitive

14 dysfunction ( $\geq 50 \%$ CBF reduction $)^{21,22}$. Thus, vascular factors beyond cerebral perfusion could

15 also be involved.

16 To address this question, we investigated whether tau contributes to the cognitive

17 impairment induced by HSD and, if so, whether the effect depends on the associated cerebral

18 hypoperfusion. First, we established if HSD induces tau phosphorylation. Male C56BI/6 mice were

19 placed on a normal diet (ND) or HSD (4 or 8\% $\mathrm{NaCl}$ ), corresponding to a 8-16 fold increase over

20 the salt content in the regular mouse chow and approaching the highest levels of human salt

21 consumption ${ }^{23}$. Phosphorylation of selected tau epitopes linked to tau aggregation and neuronal

22 dysfunction ${ }^{17}$ were assessed $4,8,12$, and 24 weeks later by Western blotting. HSD (8\%)

23 increased p-tau (AT8, RZ3 epitopes) in neocortex and hippocampus without upregulation of total

24 tau (Tau 46) (Fig. 1A). In the hippocampus, an increase in PHF13 was also observed (Extended

25 Data Fig. 1A). HSD did not increase tau acetylation, a post translational modification implicated

26 in the harmful neuronal effects of $\operatorname{tau}^{24}$ (Extended data Fig. 1A). AT8 and RZ3 were also increased

27 in neocortex of female mice fed a HSD (Extended data Fig. 1B). AT8 and MC1 immunoreactivity 
Faraco et al. / page 4

1 was detected in neuronal cell bodies of the pyriform cortex and other cortical regions, but

2 neurofibrillary tangles were not observed (Fig. 1B-C; Extended Data Fig. 1C-D). As anticipated,

3 p-tau (AT8) was abolished by incubation of the sample with lambda protein phosphatase

4 (Extended Data Fig. 1E). Increased AT8 was also observed in neocortex with a 4\% HSD

5 (Extended Data Fig. 1F), indicating that also lower amounts of dietary salt are sufficient to induce

6 tau phosphorylation.

In neocortex, the increase in AT8 was observed at 4 weeks of HSD and was greatest at 24 weeks, whereas in hippocampus p-tau peaked at 12 weeks and then declined (Fig. 1D and Extended Data Fig. 1G). An increase in RZ3 was observed at 8 and 12 weeks in neocortex and at 12 weeks in the hippocampus (Extended Data Fig. 1G). Starting at 12 weeks of HSD, mice exhibited difficulties in recognizing novel objects and developed a deficit in spatial memory at the Barnes maze, suggesting impaired cognition (Fig.1E; Extended Data Fig. 2A). Therefore, tau phosphorylation occurs in parallel with the endothelial NO deficit previously described ${ }^{8}$ and is

14 followed by cognitive deficits. To determine if p-tau is upregulated also in other conditions associated with endothelial dysfunction, we investigated models of hypertension in which deficits in endothelial NO and cognitive function are well described ${ }^{25}$. We found an increase in p-tau in

17 hypertension produced by chronic administration of the pressor peptide angiotensin-II or in $\mathrm{BPH} / 2 \mathrm{~J}$ mice with life-long elevations in blood pressure (Extended Data Fig. 2B-C).

Tau solubility is a critical determinant of its harmful neuronal effects, and insoluble tau has been implicated in the neuronal dysfunction driving cognitive impairment ${ }^{17}$. To determine whether

21 HSD alters tau solubility, tau levels were examined in neocortical and hippocampal lysates by

22 Western blotting after sequential biochemical extraction in RAB (salt buffer), RIPA (detergent

23 buffer) or $70 \%$ formic acid (FA), containing, respectively, soluble, less soluble and highly insoluble

24 tau. After 12 weeks of HSD the tau in RIPA and FA fractions increased in neocortex and 25 hippocampus, consistent with an increase in more insoluble tau (Fig. 1F-G). Since hypothermia 26 in the setting of hibernation increases p-tau levels without causing cognitive impairment ${ }^{26}$, we 27 performed a similar analysis in the brain of mice subjected to hypothermia. We found that 
Faraco et al. / page 5

1 hypothermia increases p-tau, but, at variance with HSD, does not produce a shift towards more

2 insoluble species (Extended Data Fig. 2D-E). These observations indicate that HSD leads to tau

3 phosphorylation and a shift from soluble to insoluble tau.

The NO precursor L-arginine counteracts the deficit in endothelial NO induced by HSD ${ }^{8}$

5 and other conditions associated with endothelial dysfunction ${ }^{27,28}$. Therefore, we asked if L-

6 arginine would also rescue the p-tau accumulation induced by HSD and, if so, whether the effect

7 is associated with improved cognition. To this end, mice were given L-arginine in the drinking water $(10 \mathrm{gr} / \mathrm{L})$ during the last 4 weeks of the HSD or ND 12 week-treatment. We have previously demonstrated that L-arginine normalizes resting and stimulated cerebral endothelial NO synthesis without affecting arterial pressure ${ }^{8}$. L-arginine suppressed p-tau accumulation both in neocortex (AT8 and RZ3) and hippocampus (AT8) and prevented the cognitive dysfunction induced by HSD (Fig. 2A-D; Extended Data Fig. 3A).

Cdk5 is a major kinase responsible for tau hyperphosphorylation ${ }^{29}$. Cdk5 activity is tightly regulated by its protein binding partners, including $\mathrm{p} 35^{30}$. In conditions associated with neuronal stress, cleavage of p35 into p25 by calpain leads to dysregulated activation of Cdk5 and hyperphosphorylation of its targets, including tau ${ }^{31,32}$. Since reduced endothelial NO may lead to tau phosphorylation by activating Cdk5 via p2533, we examined if HSD influences calpain and Cdk5 activity. Calpain 2 is more abundant than calpain 1 in neocortex (Fig. 3A), is located mainly in neurons (Extended Data Fig. 3B) ${ }^{34}$ and colocalizes with Cdk5 (Extended Data Fig. 3C). HSD did not alter calpain expression (Fig. 3A), but resulted in activation of the enzyme, leading to an 21 increase in the p25/p35 ratio, in Cdk5 bound to p25/p35 and in Cdk5 catalytic activity (Fig. 3A-C).

22 Attesting to the involvement of endothelial NO, L-arginine administration prevented the calpain 23 activation induced by HSD and the resulting increase in the Cdk5/p25/p35 complex and enzyme 24 activation (Fig. 3D-E). L-arginine did not alter calpain levels (Extended Data Fig. 3D). GSK3 $\beta$ has also been implicated in tau phosphorylation, but HSD did not increase the activity of this enzyme in neocortex (Extended Data Fig. 3E). Similarly, HSD did not alter the expression of the prolyl

27 cis/trans isomerase Pin-1, a regulator of tau dephosphorylation ${ }^{35}$ (Extended Data Fig. 3F). 
Faraco et al. / page 6

Next, we examined the potential mechanisms by which endothelial NO deficiency may

2 influence calpain activity. Calpain, once activated by $\mathrm{Ca}^{2+}$, is regulated mainly by its endogenous

3 inhibitor calpastatin and by nitrosylation by $\mathrm{NO}^{36}$, which suppress calpain activity ${ }^{37}$. Since HSD

4 did not reduce calpastatin expression (Extended Data Fig. 3G), we used the biotin switch assay

5 to investigate the effect of HSD on calpain nitrosylation. Consistent with the observed calpain

6 activation, we found that HSD reduces calpain nitrosylation (Fig. 3F).

The findings thus far suggest that HSD leads to neuronal p-tau accumulation through a deficit in endothelial NO resulting in denitrosylation and activation of calpain, which, in turn increases p25 levels resulting in activation of Cdk5. However, HSD also lowers resting CBF and impairs the ability of endothelial cells to regulate CBF, which could contribute to impair cognition by reducing the delivery of oxygen and glucose to brain regions involved in cognitive function ${ }^{19,38}$.

12 Therefore, we examined the relative contribution of $p$-tau and neurovascular dysfunction to the cognitive deficits induced by HSD. We reasoned that if tau is critical for the cognitive dysfunction,

14 then tau-null mice should be protected from the deleterious cognitive effects of HSD despite sustained cerebral hemodynamic dysfunction. Tau-null mice were placed on ND or HSD and cerebral endothelial vasomotor function and cognition were assessed 12 weeks later. As

17 anticipated ${ }^{39}$, the performance of tau-null mice to the novel object recognition test and Barnes maze was not different from that of WT controls fed a ND (Fig. 4A-C). Tau-null mice on HSD did not exhibit cognitive impairment, but still exhibited marked endothelial dysfunction, as reflected by the suppression of the CBF increase induced by bathing the neocortex with acetylcholine (Fig.

21 4D), a prototypical endothelial response mediated by eNOS-derived NO ${ }^{38}$. Therefore, it would 22 seem that CBF dysregulation is not required for the cognitive dysfunction of HSD. To provide

23 further evidence in support of this conclusion we treated WT mice with anti-tau antibodies (HJ8.8)

24 or control lgG (50 mg/kg/week; i.p.) for the last 4 weeks of the 12-week ND or HSD regimen ${ }^{40}$.

25 Anti-tau antibodies were previously shown to ameliorate cognitive function in a tauopathy mouse 26 model $^{40}$. In HJ8.8-treated mice, HSD induced a reduction in resting CBF and an attenuation of 27 the endothelial CBF response to acetylcholine comparable to that observed in HSD-fed mice 
Faraco et al. / page 7

1 treated with control lgG (Fig. 4E-F). Despite persistent hypoperfusion and endothelial dysfunction,

2 anti-tau antibodies ameliorated the cognitive deficit induced by HSD (Fig. 4G). The effect was

3 associated with a reduction in p-tau in neocortex (AT8) and hippocampus (AT8 and RZ3) (Fig.

4 4H). As before ${ }^{8}$, HSD did not affect the increases in CBF induced by neural activity (Extended

5 Data Fig. 4A)

6 These observations, collectively, are consistent with the hypothesis that the reduction in

7 endothelial NO induced by HSD leads to calpain denitrosylation resulting in activation of the

8 enzyme. The ensuing increase in p25 activates Cdk5 leading to tau phosphorylation in neurons,

9 which, in turn, is responsible for the cognitive impairment (Extended Data Fig. 4C). In addition,

10 the data indicate that the neurovascular dysfunction associated with HSD, also caused by the NO

11 deficit, is not critical for the cognitive dysfunction. This conclusion is supported by the observations

12 that the cognitive deficits induced by HSD do not occur in tau-null mice and are ameliorated by

13 anti-tau antibodies, despite altered endothelium-dependent vasodilatation and reduced CBF.

14 The findings provide novel insights into the mechanisms by which cerebrovascular 15 dysfunction alters cognition. Although to be confirmed in other cerebrovascular risk factors, the 16 CBF reduction and suppression of endothelium-dependent vasoreactivity associated with HSD 17 do not drive cognitive impairment. Whereas the suppression in endothelial NO induced by HSD 18 is required for the cognitive impairment, the hemodynamic consequences of such NO deficit do 19 not play a role. Rather, the cognitive dysfunction is dependent on the tau phosphorylation 20 promoted by the deficit in endothelial NO. Thus, the cerebral hypoperfusion resulting from NO 21 deficiency seems to be inconsequential to the cognitive deficit. Other aspects of endothelial 22 function are at play, namely endothelial NO maintaining calpain homeostasis and preventing Cdk5 23 dysregulation and tau hyperphosphorylation.

24 Our data also provide a previously-unrecognized link between dietary habits, vascular 25 dysfunction and tau pathology, independently of cerebral hypoperfusion. Such relationship may 26 play a role in the frequent overlap between vascular and neurogenerative pathologies underlying 27 late-life dementia ${ }^{41}$. Whereas avoiding excessive salt consumption may help prevent tau 
Faraco et al. / page 8

1 pathology, therapeutic efforts to counteract cerebrovascular dysfunction need to go beyond

2 rescuing cerebral perfusion, and target vascular mediators governing neurovascular interactions

3 critical for cognitive health.

4

\section{MATERIALS AND METHODS}

6 Most of the methods used in this study are well established in the laboratory and have been

7 described in detail in previous publications $8,25,42$. Here we provide only a brief description.

$9 \quad$ Mice

10 All procedures are approved by the institutional animal care and use committee of Weill Cornell

11 Medicine (Animal protocol number: 0807-777A). Studies were conducted, according to the

12 ARRIVE guidelines (https://www.nc3rs.org.uk/arrive-guidelines), in the following lines of mice:

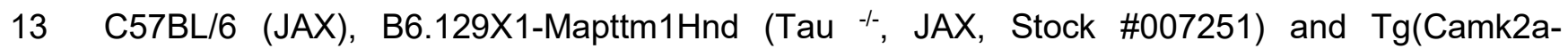

14 tTA)1Mmay Fgf ${ }^{14 T g\left(t e t O-M A P T^{*} \text { 301L)4510Kha/J }(r T g 4510, ~ J A X, ~ S t o c k \# 024854), ~ B P H / 2 J ~ m i c e ~(A g e: ~\right.} 5$

15 months; JAX, Stock \#003005) and BPN/3J mice (Age: 5 months; JAX, Stock \#003004). Unless

16 otherwise indicated, male mice were used.

18 High Salt Diet

19 Male or female mice (8 weeks old) received normal chow $(0.5 \% \mathrm{NaCl})$ and tap water ad libitum

20 (normal diet) or sodium-rich chow (4-8\% $\mathrm{NaCl})$ and tap water containing $1 \% \mathrm{NaCl}$ ad libitum

21 (HSD) for 4 to 24 weeks according to the experiment ${ }^{8}$.

\section{In vivo treatments}

24 The nitric oxide precursor, L-arginine (10gr/L; Sigma) was administered in the drinking water

25 starting at 8 weeks of HSD and continuing until 12 weeks. ND and HSD mice were treated (i.p., 26 weekly) with $50 \mathrm{mg} / \mathrm{kg}$ of anti-Tau (HJ8.8) or mouse lgG1 isotype control (Clone MOPC-21; 
Faraco et al. / page 9

1 bioXcell) antibodies for the last 4 weeks of the HSD treatment period (12 weeks) prior to

2 behavioral and cerebrovascular studies.

3

4 General surgical procedures for CBF studies

5 Mice were anesthetized with isoflurane (induction, 5\%; maintenance, $2 \%$ ). The trachea was

6 intubated and mice were artificially ventilated with a mixture of $\mathrm{N}_{2}$ and $\mathrm{O}_{2}$. One of the femoral

7 arteries was cannulated for recording mean arterial pressure (MAP) and collecting blood samples

8 for blood gas analysis ${ }^{43}$. Rectal temperature was maintained at $37^{\circ} \mathrm{C}$. End tidal $\mathrm{CO}_{2}$, monitored

9 by a $\mathrm{CO}_{2}$ analyzer (Capstar-100, CWE Inc.), was maintained at 2.6-2.7\% to provide a $\mathrm{pCO}_{2}$ of

$10 \quad 30-40 \mathrm{mmHg}$ and a $\mathrm{pH}$ of 7.3-7.437. After surgery, isoflurane was discontinued and anesthesia

11 was maintained with urethane (750 mg/kg, i.p.) and chloralose (50 mg/kg, i.p.). Throughout the

12 experiment the level of anesthesia was monitored by testing motor responses to tail pinch.

\section{Monitoring cerebral blood flow}

15 A small craniotomy $(2 \times 2 \mathrm{~mm})$ was performed to expose the parietal cortex, the dura was removed,

16 and the site was superfused with Ringer's solution $\left(37^{\circ} \mathrm{C} ; \mathrm{pH} 7.3-7.4\right)^{25}$. CBF was continuously

17 monitored at the site of superfusion with a laser-Doppler probe (Perimed) positioned

18 stereotaxically $\approx 0.5 \mathrm{~mm}$ above the cortical surface and connected to a data acquisition system

19 (PowerLab). CBF values are expressed as percentage increases relative to the resting level.

\section{Protocol for CBF experiments}

22 After MAP and blood gases stabilized, CBF responses were recorded ${ }^{8}$. The whisker-barrel cortex

23 was activated for 60 seconds by stroking the contralateral vibrissae, and the evoked changes in

24 CBF were recorded. The endothelium-dependent vasodilator acetylcholine (ACh; 100 $\mu$ M; Sigma),

25 was superfused on the exposed neocortex for 5 minutes and the associated CBF changes were 26 recorded by laser-Doppler flowmetry. 
Faraco et al. / page10

\section{Measurement of resting CBF by ASL-MRI}

2 CBF was assessed quantitatively using arterial spin labeling magnetic resonance imaging (ASL-

$3 \mathrm{MRI}$ ) as previously described ${ }^{8}$. The ASL images were analyzed by ImageJ and the average CBF

4 value is reported as $\mathrm{mL}$ per $100 \mathrm{~g}$ of tissue per minute.

6 Osmotic minipumps implantation

7 Osmotic minipumps containing vehicle (saline) or ANG II (600 $\mathrm{ng} \cdot \mathrm{kg}-1 \cdot \mathrm{min}-1)$ were implanted

8 subcutaneously under isoflurane anesthesia. Systolic blood pressure was monitored in awake

9 mice using tail-cuff plethysmography ${ }^{25}$. Forty-two days later, mice were anesthetized and their brains were collected for assessment of tau phosphorylation.

\section{Hypothermia}

13 C57BL/6 mice (12 weeks old) were anesthetized by injection of ketamine/xylazine (100/10

$14 \mathrm{mg} / \mathrm{kg}$ ). Rectal temperature was continuously monitored and kept at $37^{\circ} \mathrm{C}$ (normothermia) or $30^{\circ} \mathrm{C}$ 15 (hypothermia) using a thermostatically-controlled heating pad. Mice were sacrificed 30 minutes 16 after anesthesia and their brains were collected and frozen on dry ice. Tissues were kept at $-80^{\circ} \mathrm{C}$ 17 until processing for immunoblot analysis.

\section{Immunoblot analysis}

20 Cortex $(\approx 80-90 \mathrm{mg})$ and hippocampus $(\approx 15 \mathrm{mg})$ isolated from ND and HSD mice were sonicated

21 in 800 and $600 \mu$ l of RIPA buffer $(50 \mathrm{mM}$ Tris- $\mathrm{HCl} \mathrm{pH} 8.0,150 \mathrm{mM} \mathrm{NaCl}, 0.5 \%$ Deoxycholic Acid,

$220.1 \%$ SDS, 1mM EDTA pH 8.0, 1\% IGEPAL CA-630, 1mM Na3VO4, 20mM NaF and one

23 tablet $/ 10 \mathrm{~mL}$ of cOmplete ${ }^{\mathrm{TM}}$, EDTA-free Protease Inhibitor Cocktail, Millipore Sigma) and equal

24 volumes were mixed with SDS sample buffer, boiled, and analyzed on $10 \%$ or $10-20 \%$ Novex $^{\mathrm{TM}}$

25 WedgeWell ${ }^{\mathrm{TM}}$ gels (Thermo Fisher Scientific). Proteins were transferred to PVDF membranes

26 (Millipore), blocked at room temperature (RT) for 1 hour with $5 \%$ milk in TBS, and incubated,

27 overnight at $4^{\circ} \mathrm{C}$, with primary antibodies (see Extended Data Table 1 ) in $5 \%$ BSA in TBS/0.1\% 
Faraco et al. / page11

1 Tween-20 (TBST). Membranes were washed in TBST, incubated with goat anti-mouse or rabbit

2 secondary antibodies conjugated to horseradish peroxidase (Santa Cruz Biotechnology) for 1

3 hour at RT and protein bands were visualized with Clarity Western ECL Substrate (Bio Rad) on a

4 Bio Rad ChemiDoc MP Imaging System. Quantification was performed using Image Lab 6.0 (Bio

5 Rad).

6

$7 \quad$ Heat-stable fractions

8 After homogenization in cold RIPA buffer and centrifugation, $150 \mu$ l of the supernatant containing

9 the proteins was boiled at $100^{\circ} \mathrm{C}$ for 10 minutes. Samples were cooled on ice for 20 minutes and

10 then centrifuged at $20,000 \mathrm{~g}$ at $4^{\circ} \mathrm{C}$ for 15 minutes. The supernatant corresponding to the heat

11 stable (HS) fraction was then harvested. This method is used to isolate proteins resistant to heat

12 including tau and other microtubule-associated proteins (MAPs). Thus, endogenous

13 immunoglobulins are precipitated during the boiling process and eliminated from the supernatant.

14 The proteins were then mixed with equal volumes of SDS sample buffer, boiled, and analyzed on

$1510 \%$ Novex $^{\mathrm{TM}}$ WedgeWell ${ }^{\mathrm{TM}}$ gels (Thermo Fisher Scientific). Although Tau protein is partially lost

16 during the boiling process, the HS samples are enriched with Tau (please see Extended Data Fig.

17 4B). Furthermore, boiling significantly improves specificity of certain antibodies such as AT8, RZ3

18 or $\mathrm{MC}^{44}$.

\section{Tau dephosphorylation}

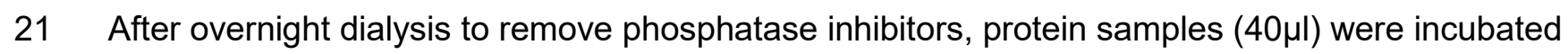
22 with $5 \mu \mathrm{L}$ of $10 \mathrm{X}$ NEBuffer for Protein MetalloPhosphatases (PMP), $5 \mu \mathrm{L}$ of $10 \mathrm{mM} \mathrm{MnCl}_{2}$ and $1 \mu \mathrm{l}$ 23 of Lambda Protein Phosphatase (Lambda PP, New England Biolabs) at $30^{\circ} \mathrm{C}$ for 3 hours.

24 Reactions were stopped by addition of SDS sample buffer and boiling for 5 minutes at $100{ }^{\circ} \mathrm{C}$. 
Faraco et al. / page 12

1 Extraction was performed as described previously ${ }^{40}$. The cortex $(\approx 80-90 \mathrm{mg})$ and the

2 hippocampus $(\approx 15 \mathrm{mg})$ of each brain were homogenized by sonication in 800 and $300 \mu$ of $\mathrm{RAB}$

3 buffer [100mM MES, 1mM EDTA, 0.5mM MgSO $4,750 \mathrm{mM} \mathrm{NaCl,} \mathrm{20mM} \mathrm{NaF,} \mathrm{1mM} \mathrm{Na3VO4,}$

4 supplemented by EDTA-free Protease Inhibitor Cocktail, Millipore Sigma], respectively. In brief,

5 the samples were centrifuged at $50,000 \mathrm{~g}$ for 20 minutes at $4^{\circ} \mathrm{C}$ using an Optima MAX-TLA 120.2

6 Ultracentrifuge (Beckman). The supernatants were collected as RAB soluble fractions and pellets

7 were resuspended in identical volumes of RIPA buffer $[150 \mathrm{mM} \mathrm{NaCl}, 50 \mathrm{mM}$ Tris, $0.5 \%$

8 deoxycholic acid, $1 \%$ Triton X-100, 0.5\% SDS, 25mM EDTA, pH 8.0, 20mM NaF, $1 \mathrm{mM} \mathrm{Na}_{3} \mathrm{VO}_{4}$

9 supplemented by EDTA-free Protease Inhibitor Cocktail, Millipore Sigma], and centrifuged at

$1050,000 \mathrm{~g}$ for 20 minutes at $4^{\circ} \mathrm{C}$. The supernatants were collected as RIPA soluble fractions. The

11 pellets were sonicated in $70 \%$ formic acid $(300 \mu$ l for the cortex and $125 \mu$ f for the hippocampus),

12 and centrifuged at $50,000 \mathrm{~g}$ for 20 minutes at $4^{\circ} \mathrm{C}$. The supernatants were collected as $70 \%$ formic

13 acid fractions. All fractions were stored in $-80^{\circ} \mathrm{C}$ until analyzed. For western blotting, an aliquot of

$14100 \mu \mathrm{l}$ of the formic acid fractions was evaporated in a Savant SpeedVac concentrator at $45^{\circ} \mathrm{C}$ for

151 hour. The samples were resuspended in $100 \mu$ l of SDS sample buffer with the addition of $1 \mu$ l of

$1610 \mathrm{~N} \mathrm{NaOH}$, sonicated and then boiled for 5 minutes.

Immunohistochemistry

19 After 12 weeks of ND/HSD, mice were anesthetized with intraperitoneal pentobarbital (200

$20 \mathrm{mg} / \mathrm{kg}$ ), and then perfused transcardiacally with cold PBS, followed by cold 4\% paraformaldehyde

21 (PFA) in PBS. The brains were removed and immersed first in 4\% PFA overnight and then in 70\%

22 ethanol for 3 days. Brains were then embedded in paraffin and cut into $6 \mu \mathrm{m}$ sections using a

23 microtome. After rehydration and antigen retrieval in preheated citrate buffer (10 $\mu \mathrm{M})$ for 30

24 minutes, brain sections were immersed in $3 \% \mathrm{H}_{2} \mathrm{O}_{2}$ and then blocked with $100 \%$ Sniper (Biocare

25 Medical) for 1 hour. After blocking, sections were incubated for 2.5 days at $4^{\circ} \mathrm{C}$ with the AT8,

26 MC1, Calpain 2 or Cdk5 antibody $(1: 250,1: 100,1: 100$ and 1:100 in 1:50 Sniper in PBS,

27 respectively) and thereafter processed for 1 hour with the biotinylated secondary antibody in $1 \%$ 
Faraco et al. / page13

1 normal donkey serum PBS (anti-mouse IgG1, Jackson ImmunoResearch) or Cy3 anti-rabbit and

2 FITC anti-mouse (Jackson ImmunoResearch) for immunofluorescence studies. Reactions were

3 visualized with the ABC-complex (Vectorlabs) and 3,3-diaminobenzidine. A Nikon light

4 microscope or a confocal microscope (Leica TCS SP5) was used to visualize the signal

5 associated with each antibody.

6

$7 \quad$ Thioflavin S Staining

8 After mounting on slides and post-fixation with 4\% PFA in PBS for 10 minutes, coronal brain

9 sections $(40 \mu \mathrm{m})$ were washed and labeled with $0.05 \%$ (wt/vol) thioflavine-S in $50 \%$ (vol/vol)

10 ethanol for 10 minutes as previously described ${ }^{45}$. An epifluorescence microscope (IX83 Inverted

11 Microscope, Olympus) was used to visualize the FITC signal associated with thioflavine-S.

\section{Calpain activity}

14 Calpain activity was measured by using a Calpain Activity Assay Kit from AbCam ${ }^{46,47}$. Briefly, 15 fresh cortex and hippocampus were homogenized in the extraction buffer provided with the kit, 16 which specifically extracts cytosolic proteins without contaminations of cell membrane and 17 lysosome proteases and prevents auto-activation of calpain during the extraction procedure. The 18 fluorometric assay is based on the detection of cleavage of calpain substrate Ac-LLY-AFC. Ac19 LLY-AFC emits blue light $(\Lambda \max =400 \mathrm{~nm})$; upon cleavage of the substrate by calpain, free AFC 20 emits a yellow-green fluorescence $(\wedge \max =505 \mathrm{~nm})$, which can be quantified using a fluorometer 21 or a fluorescence plate reader. Specificity of the signal was confirmed by using the calpain

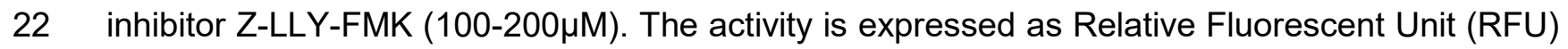
23 per milligram of protein for each sample.

25 p35/p25 and GSK3 $\beta$ immunoprecipitation

26 Immunoprecipitation was performed with anti-p35p25 (Cell Signaling), anti-GSK3 $\beta$ (Cell 27 Signaling) or anti-rabbit monoclonal lgG1 isotype control antibody (Santa Cruz Biotechnology). 
Faraco et al. / page14

1 Samples were incubated overnight with the primary antibodies and then with protein-A sepharose

2 (p35p25) (GE Healthcare Life Sciences) or protein-G Dynabeads (GSK3 $\beta$ ) (Thermo Fisher

3 Scientific) for 2 hours at $4^{\circ} \mathrm{C}$. Precipitates were used for Cdk5 or GSK3 $\beta$ activity measurements.

4 Immunoprecipitation was confirmed by loading the samples on $10 \%$ Tris-glycine SDS

5 polyacrylamide gels and western blot as described above.

6

\section{Detection of S-nitrosylation of calpain 2 with the biotin-switch technique}

Detection of S-nitrosylated calpain 2 was performed using the biotin-switch technique, as previously described ${ }^{48}$. Briefly, samples were sonicated in $800 \mu$ l of RIPA buffer containing $0.1 \mathrm{mM}$ of neocuproine and, after centrifugation, protein concentrations were measured. Cysteine thiol groups in $1 \mathrm{mg}$ of proteins were blocked with $10 \%$ S-methylmethane thiosulfonate (MMTS) (Sigma). After protein-precipitation with $100 \%$ acetone, sodium ascorbate was added to the sample to convert each S-nitrothiols (SNO) to a free thiol via a transnitrosation reaction to generate O-nitrosoascorbate. Next, each nascent free thiol (previously an SNO site) was biotinylated with biotin-HPDP (Pierce). Biotinylated proteins were then pull-down by using avidin beads and analyzed on $10 \%$ Novex $^{\mathrm{TM}}$ WedgeWell $^{\mathrm{TM}}$ gels (Thermo Fisher Scientific). Before avidin pulldown, a small fraction of each sample was collected to determine protein "input." The degree of pulldown correlates with protein S-nitrosylation of calpain 2 which was detected with an antibody against the protein (see Extended Data Suppl. Table 1). Nitrosylation of calpain 2 is expressed as the ratio between the pull-down signal and the input corrected for the $\beta$-actin levels.

\section{Cdk5 and GSK3 $\beta$ activity}

Cdk5 activity in brain lysates was determined after pulldown with p25/p35 antibody (Cell Signaling) from $500 \mu \mathrm{g}$ total protein using a synthetic histone $\mathrm{H} 1$ peptide substrate (PKTPKKAKKL, Enzo Life Sciences). GSK3 $\beta$ activity was determined after pulldown with GSK3 $\beta$

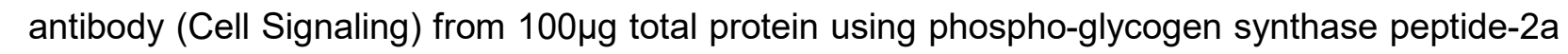
as substrate (Tocris). Phosphorylation reactions were initiated by mixing bead-coupled Cdk5 with 
Faraco et al. / page15

$140 \mu \mathrm{l}$ reaction buffer containing the following: 50mM HEPES.KOH (pH 7.4), $5 \mathrm{mM} \mathrm{MgCl}, 0.05 \%$

$2 \mathrm{BSA}, 50 \mu \mathrm{M}$ substrate, $50 \mu \mathrm{M}$ cold ATP, $1 \mathrm{mM}$ dithiothreitol, $1 \mathrm{x}$ complete protease inhibitors without

3 EDTA (Roche Applied Biosciences) and $5 \mathrm{Ci} / \mathrm{mmole}{ }^{{ }^{3} 2} \mathrm{P}-\mathrm{ATP}$. Companion reactions for every

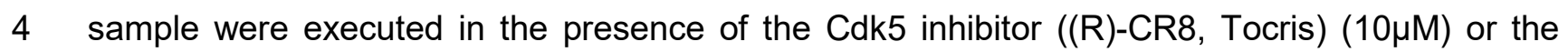

5 GSK3 $\beta$ inhibitor (CHIR 99021, Tocris) $(10 \mu \mathrm{M})$ to correct for non-specific activity. Reactions were

6 incubated at $30^{\circ} \mathrm{C}$ for 30 minutes, after which they were terminated by spotting on P81

7 phosphocellulose cation exchange chromatography paper. Filters were washed 4 times for 2

8 minutes in $0.5 \%$ phosphoric acid, and the remaining radioactivity was quantified in a scintillation

9 counter by the Cherenkov method.

11 Novel Object Recognition Test

12 The novel object recognition test (NOR) task was conducted under dim light in a plastic box.

13 Stimuli consisted of plastic objects that varied in color and shape but had similar size $\mathrm{e}^{49,50}$. A video

14 camera mounted on the wall directly above the box was used to record the testing session for off-

15 line analysis. Mice were acclimated to the testing room and chamber for one day prior to testing.

16 Twenty-four hours after habituation, mice were placed in the same box in the presence of two

17 identical sample objects and were allowed to explore for 5 minutes. After an intersession interval

18 of 1 hour, mice were placed in the same box but one of the two objects was replaced by a novel

19 object. Mice were allowed to explore for 5 minutes. Exploratory behavior was later assessed

20 manually by an experimenter blinded to the treatment group. Exploration of an object was defined

21 as the mouse sniffing the object or touching the object while looking at it. Placing the forepaws on

22 the objects was considered as exploratory behavior but climbing on the objects was not. A minimal

23 exploration time for both objects (total exploration time) during the test phase ( $\sim 5$ seconds) was

24 used. The amount of time taken to explore the novel object was expressed as percentage of the 25 total exploration time and provides an index of recognition memory ${ }^{49,50}$.

\section{The Barnes Maze test}


Faraco et al. / page 16

1 The Barnes maze consisted of a circular open surface $(90 \mathrm{~cm}$ in diameter) elevated to $90 \mathrm{~cm}$ by

2 four wooden legs ${ }^{51}$. There were 20 circular holes $(5 \mathrm{~cm}$ in diameter) equally spaced around the

3 perimeter, and positioned $2.5 \mathrm{~cm}$ from the edge of the maze. No wall and no intra-maze visual

4 cues were placed around the edge. A wooden plastic escape box $(11 \times 6 \times 5 \mathrm{~cm})$ was positioned

5 beneath one of the holes. Two neon lamps and a buzzer were used as aversive stimuli. The Any-

6 Maze tracking system (Stoelting) was used to record the movement of mice on the maze. Extra-

7 maze visual cues consisted of objects within the room (table, computer, sink, door, etc.) and the

8 experimenter. Mice were tested in groups of seven to ten, and between trials they were placed

9 into cages, which were placed in a dark room adjacent to the test room for the inter-trial interval

10 (20-30 minutes). No habituation trial was performed. The acquisition phase consisted of 3

11 consecutive training days with three trials per day with the escape hole located at the same

12 location across trials and days. On each trial a mouse was placed into a start tube located in the

13 center of the maze, the start tube was raised, and the buzzer was turned on until the mouse

14 entered the escape hole. After each trial, mice remained in the escape box for 60 seconds before

15 being returned to their cage. Between trials the maze floor was cleaned with $10 \%$ ethanol in water

16 to minimize olfactory cues. For each trial mice were given 3 minutes to locate the escape hole,

17 after which they were guided to the escape hole or placed directly into the escape box if they

18 failed to enter the escape hole. Four parameters of learning performance were recorded: (1) the

19 latency to locate (primary latency) and (2) enter the escape hole (total latency), (3) the number of

20 errors made and (4) the distance traveled before locating the escape hole ${ }^{51}$. When a mouse

21 dipped its head into a hole that did not provide escape was considered an error. On days 4 and

225 , the location of the escape hole was moved $180^{\circ}$ from its previous location (reverse learning)

23 and two trials per day were performed.

\section{Statistics}

26 Sample size was determined according to power analysis based on previously published work by

27 our lab on the effects of dietary salt on CBF regulation and cognitive function. On these bases, 
Faraco et al. / page17

$1 \quad 10-15$ mice/group were required in studies involving assessment of cognitive function and

2 cerebrovascular function ${ }^{8,25}$. Mouse randomization was performed based on the random number

3 generator function (RANDBETWEEN) in Microsoft Excel software. Analysis of the data was

4 performed in a blinded fashion and GraphPad Prism (v. 6.0) software was used for statistical

5 analysis. Intergroup differences were analyzed by unpaired Student's t-test for single comparison

6 or by one or two-way analysis of variance (Tukey's or Bonferroni's post-hoc analysis) for multiple

7 comparisons. Data are expressed as mean \pm SEM and differences are considered statistically

8 significant for $p<0.05$.

10 Data Availability

11 All data generated or analyzed during this study are included in this published article (and its 12 supplementary information files).

\section{ACKNOWLEDGEMENTS}

15 We thank Prof. Peter Davies for providing the RZ3, MC1 and PHF1 antibodies. This study was 16 supported by National Institutes of Health grants R37-NS089323 (Cl) and 1R01-NS095441 (Cl), 17 by a grant from the Cure Alzheimer's Fund (GF and Cl), and by a Scientist Development Grant 18 from the American Heart Association (GF). The support from the Feil Family Foundation is 19 gratefully acknowledged.

\section{AUTHOR CONTRIBUTIONS}

22 G.F. performed western blotting experiments, behavioral tests, cerebrovascular studies and 23 analyzed data. K.H. performed experiments on Cdk5/GSK3 $\beta$ activity and analyzed data. S.G.S.

24 performed western blotting experiments, behavioral tests and immunohistochemistry. S.S. and 25 M.M.S. performed experiments on the effects of hypertension on tau. A.M. performed 26 immunohistochemistry experiments. H.J. and D.M.H. provided the HJ8.8 antibody. J. A 
Faraco et al. / page 18 supervised the molecular aspects of the study and edited the manuscript. G.F. and C.I. designed and supervised the entire study and wrote the manuscript.

3

\section{COMPETING INTERESTS}

D.M.H. is listed as an inventor on a patent licensed by Washington University to C2N Diagnostics

and subsequently AbbVie on the therapeutic use of anti-tau antibodies. D.M.H. co-founded and

is on the scientific advisory board of C2N Diagnostics. D.M.H. is on the scientific advisory board of Denali, Genentech, and Proclara.

\section{REFERENCES}

1 Scarmeas, N., Anastasiou, C. A. \& Yannakoulia, M. Nutrition and prevention of cognitive impairment. Lancet Neurol, doi:10.1016/S1474-4422(18)30338-7 (2018).

2 van de Rest, O., Berendsen, A. A., Haveman-Nies, A. \& de Groot, L. C. Dietary patterns, cognitive decline, and dementia: a systematic review. Adv Nutr 6, 154-168, doi:10.3945/an.114.007617 (2015).

3 Voortman, T. et al. Adherence to the 2015 Dutch dietary guidelines and risk of noncommunicable diseases and mortality in the Rotterdam Study. Eur J Epidemiol 32, 9931005, doi:10.1007/s10654-017-0295-2 (2017).

$4 \quad$ Raz, L. et al. Hypoxia promotes tau hyperphosphorylation with associated neuropathology in vascular dysfunction. Neurobiol Dis, doi:10.1016/j.nbd.2018.07.009 (2018).

5 Qiu, L. et al. Chronic cerebral hypoperfusion enhances Tau hyperphosphorylation and reduces autophagy in Alzheimer's disease mice. Sci Rep 6, 23964, doi:10.1038/srep23964 (2016).

6 Nation, D. A. et al. Pulse pressure in relation to tau-mediated neurodegeneration, cerebral amyloidosis, and progression to dementia in very old adults. JAMA Neurol 72 , 546-553, doi:10.1001/jamaneurol.2014.4477 (2015).

7 Kim, H. J. et al. Assessment of Extent and Role of Tau in Subcortical Vascular Cognitive Impairment Using 18F-AV1451 Positron Emission Tomography Imaging. JAMA Neurol 75, 999-1007, doi:10.1001/jamaneurol.2018.0975 (2018).

8 Faraco, G. et al. Dietary salt promotes neurovascular and cognitive dysfunction through a gut-initiated TH17 response. Nat Neurosci 21, 240-249, doi:10.1038/s41593-0170059-z (2018).

9 Farquhar, W. B., Edwards, D. G., Jurkovitz, C. T. \& Weintraub, W. S. Dietary sodium and health: more than just blood pressure. J Am Coll Cardiol 65, 1042-1050, doi:10.1016/j.jacc.2014.12.039 (2015).

10 Strazzullo, P., D'Elia, L., Kandala, N. B. \& Cappuccio, F. P. Salt intake, stroke, and cardiovascular disease: meta-analysis of prospective studies. BMJ 339, b4567, doi:10.1136/bmj.b4567 (2009).

11 Fiocco, A. J. et al. Sodium intake and physical activity impact cognitive maintenance in older adults: the NuAge Study. Neurobiol Aging 33, 829 e821-828, doi:10.1016/j.neurobiolaging.2011.07.004 (2012).

12 Gardener, H., Rundek, T., Wright, C. B., Elkind, M. S. \& Sacco, R. L. Dietary sodium and risk of stroke in the Northern Manhattan study. Stroke 43, 1200-1205, doi:10.1161/STROKEAHA.111.641043 (2012). 
Faraco et al. / page19
Makin, S. D. J. et al. Small Vessel Disease and Dietary Salt Intake: Cross-Sectional Study and Systematic Review. J Stroke Cerebrovasc Dis 26, 3020-3028,
14 Heye, A. K. et al. Blood pressure and sodium: Association with MRI markers in cerebral small vessel disease. J Cereb Blood Flow Metab 36, 264-274,
15 ladecola, C. The pathobiology of vascular dementia. Neuron 80, 844-866, doi:10.1016/j.neuron.2013.10.008 (2013).
16 Scheltens, P. et al. Alzheimer's disease. Lancet 388, 505-517, doi:10.1016/S0140-
17 Wang, Y. \& Mandelkow, E. Tau in physiology and pathology. Nat Rev Neurosci 17, 5-21, doi:10.1038/nrn.2015.1 (2016).
18 Despres, C. et al. Identification of the Tau phosphorylation pattern that drives its aggregation. Proc Natl Acad Sci U S A 114, 9080-9085, doi:10.1073/pnas.1708448114 (2017).
19 Sweeney, M. D., Kisler, K., Montagne, A., Toga, A. W. \& Zlokovic, B. V. The role of brain vasculature in neurodegenerative disorders. Nat Neurosci 21, 1318-1331, doi:10.1038/s41593-018-0234-x (2018).
20 Shi, Y. et al. Cerebral blood flow in small vessel disease: A systematic review and meta- analysis. J Cereb Blood Flow Metab 36, 1653-1667, doi:10.1177/0271678X16662891 (2016).
21 Tatemichi, T. K., Desmond, D. W., Prohovnik, I. \& Eidelberg, D. Dementia associated with bilateral carotid occlusions: neuropsychological and haemodynamic course after extracranial to intracranial bypass surgery. J Neurol Neurosurg Psychiatry 58, 633-636 (1995).
22 Marshall, R. S. et al. Cerebral hemodynamics and cognitive impairment: baseline data from the RECON trial. Neurology 78, 250-255, doi:10.1212/WNL.0b013e31824365d3 (2012).
23 Powles, J. et al. Global, regional and national sodium intakes in 1990 and 2010: a systematic analysis of $24 \mathrm{~h}$ urinary sodium excretion and dietary surveys worldwide. BMJ Open 3, e003733, doi:10.1136/bmjopen-2013-003733 (2013).
24 Min, S. W. et al. Critical role of acetylation in tau-mediated neurodegeneration and cognitive deficits. Nat Med 21, 1154-1162, doi:10.1038/nm.3951 (2015).
25 Faraco, G. et al. Perivascular macrophages mediate the neurovascular and cognitive dysfunction associated with hypertension. J Clin Invest 126, 4674-4689, doi:10.1172/JCI86950 (2016).
26 Arendt, T., Stieler, J. T. \& Holzer, M. Tau and tauopathies. Brain Res Bull 126, 238-292, doi:10.1016/j.brainresbull.2016.08.018 (2016).
27 Rosenblum, W. I., Nelson, G. H. \& Shimizu, T. L-arginine suffusion restores response to acetylcholine in brain arterioles with damaged endothelium. Am J Physiol 262, H961- 964, doi:10.1152/ajpheart.1992.262.4.H961 (1992).
28 Yamada, M. et al. Endothelial nitric oxide synthase-dependent cerebral blood flow augmentation by L-arginine after chronic statin treatment. J Cereb Blood Flow Metab 20, 709-717, doi:10.1097/00004647-200004000-00008 (2000).
29 Kimura, T., Ishiguro, K. \& Hisanaga, S. Physiological and pathological phosphorylation of tau by Cdk5. Front Mol Neurosci 7, 65, doi:10.3389/fnmol.2014.00065 (2014).
30 Tsai, L. H., Delalle, I., Caviness, V. S., Jr., Chae, T. \& Harlow, E. p35 is a neural-specific regulatory subunit of cyclin-dependent kinase 5. Nature 371, 419-423, doi:10.1038/371419a0 (1994).
31 Lee, M. S. et al. Neurotoxicity induces cleavage of p35 to p25 by calpain. Nature 405, 360-364, doi:10.1038/35012636 (2000).
32 Patrick, G. N. et al. Conversion of p35 to p25 deregulates Cdk5 activity and promotes neurodegeneration. Nature 402, 615-622, doi:10.1038/45159 (1999). 
Faraco et al. / page20
Austin, S. A. \& Katusic, Z. S. Loss of Endothelial Nitric Oxide Synthase Promotes p25 Generation and Tau Phosphorylation in a Murine Model of Alzheimer's Disease. Circ Res 119, 1128-1134, doi:10.1161/CIRCRESAHA.116.309686 (2016).
34 Adamec, E., Mohan, P., Vonsattel, J. P. \& Nixon, R. A. Calpain activation in neurodegenerative diseases: confocal immunofluorescence study with antibodies specifically recognizing the active form of calpain 2. Acta Neuropathol 104, 92-104, doi:10.1007/s00401-002-0528-6 (2002).
35 Kimura, T. et al. Isomerase Pin1 stimulates dephosphorylation of tau protein at cyclin- dependent kinase (Cdk5)-dependent Alzheimer phosphorylation sites. J Biol Chem 288, 7968-7977, doi:10.1074/jbc.M112.433326 (2013).
36 Ono, Y., Saido, T. C. \& Sorimachi, H. Calpain research for drug discovery: challenges and potential. Nat Rev Drug Discov 15, 854-876, doi:10.1038/nrd.2016.212 (2016). Calcium-Dependent Protease Calpain in the Endothelial Dysfunction Induced by MPO (Myeloperoxidase). Hypertension 71, 761-770,
37 Etwebi, Z., Landesberg, G., Preston, K., Eguchi, S. \& Scalia, R. Mechanistic Role of the doi:10.1161/HYPERTENSIONAHA.117.10305 (2018).
38 ladecola, C. The Neurovascular Unit Coming of Age: A Journey through Neurovascular Coupling in Health and Disease. Neuron 96, 17-42, doi:10.1016/j.neuron.2017.07.030 (2017).
39 Ahmed, T. et al. Cognition and hippocampal synaptic plasticity in mice with a homozygous tau deletion. Neurobiol Aging 35, 2474-2478, doi:10.1016/j.neurobiolaging.2014.05.005 (2014).
40 Yanamandra, K. et al. Anti-tau antibodies that block tau aggregate seeding in vitro markedly decrease pathology and improve cognition in vivo. Neuron 80, 402-414, doi:10.1016/j.neuron.2013.07.046 (2013).
41 Power, M. C. et al. Combined neuropathological pathways account for age-related risk of dementia. Ann Neurol 84, 10-22, doi:10.1002/ana.25246 (2018).
42 Hochrainer, K. et al. The ubiquitin ligase HERC3 attenuates NF-kappaB-dependent transcription independently of its enzymatic activity by delivering the RelA subunit for degradation. Nucleic Acids Res 43, 9889-9904, doi:10.1093/nar/gkv1064 (2015).
43 Faraco, G. et al. Circulating endothelin-1 alters critical mechanisms regulating cerebral microcirculation. Hypertension 62, 759-766, doi:10.1161/HYPERTENSIONAHA.113.01761 (2013).
44 Petry, F. R. et al. Specificity of anti-tau antibodies when analyzing mice models of Alzheimer's disease: problems and solutions. PLoS One 9, e94251, doi:10.1371/journal.pone.0094251 (2014).
45 Faraco, G. et al. Hypertension enhances Abeta-induced neurovascular dysfunction, promotes beta-secretase activity, and leads to amyloidogenic processing of APP. $J$ Cereb Blood Flow Metab 36, 241-252, doi:10.1038/jcbfm.2015.79 (2016).
46 Voit, A. et al. Reducing sarcolipin expression mitigates Duchenne muscular dystrophy and associated cardiomyopathy in mice. Nat Commun 8, 1068, doi:10.1038/s41467-017- 01146-7 (2017).
47 Liu, W. et al. Metabolic stress-induced cardiomyopathy is caused by mitochondrial dysfunction due to attenuated Erk5 signaling. Nat Commun 8, 494, doi:10.1038/s41467- 017-00664-8 (2017).
48 Forrester, M. T., Foster, M. W., Benhar, M. \& Stamler, J. S. Detection of protein S- nitrosylation with the biotin-switch technique. Free Radic Biol Med 46, 119-126, doi:10.1016/j.freeradbiomed.2008.09.034 (2009).
49 Cohen, S. J. \& Stackman, R. W., Jr. Assessing rodent hippocampal involvement in the novel object recognition task. A review. Behav Brain Res 285, 105-117, doi:10.1016/j.bbr.2014.08.002 (2015).
50 Grayson, B. et al. Assessment of disease-related cognitive impairments using the novel object recognition (NOR) task in rodents. Behav Brain Res 285, 176-193, doi:10.1016/j.bbr.2014.10.025 (2015). 
bioRxiv preprint doi: https://doi.org/10.1101/470666; this version posted November 14, 2018. The copyright holder for this preprint (which was not certified by peer review) is the author/funder. All rights reserved. No reuse allowed without permission.

Faraco et al. / page21

$151 \quad$ O'Leary, T. P. \& Brown, R. E. Optimization of apparatus design and behavioral

2

3

4 measures for the assessment of visuo-spatial learning and memory of mice on the Barnes maze. Learn Mem 20, 85-96, doi:10.1101/lm.028076.112 (2013). 
bioRxiv preprint doi: https://doi.org/10.1101/470666; this version posted November 14, 2018. The copyright holder for this preprint (which was

A notcertified by peer rejpsw) is the author/funder. All rights reserved. Afg reuse allowed withzut permission.
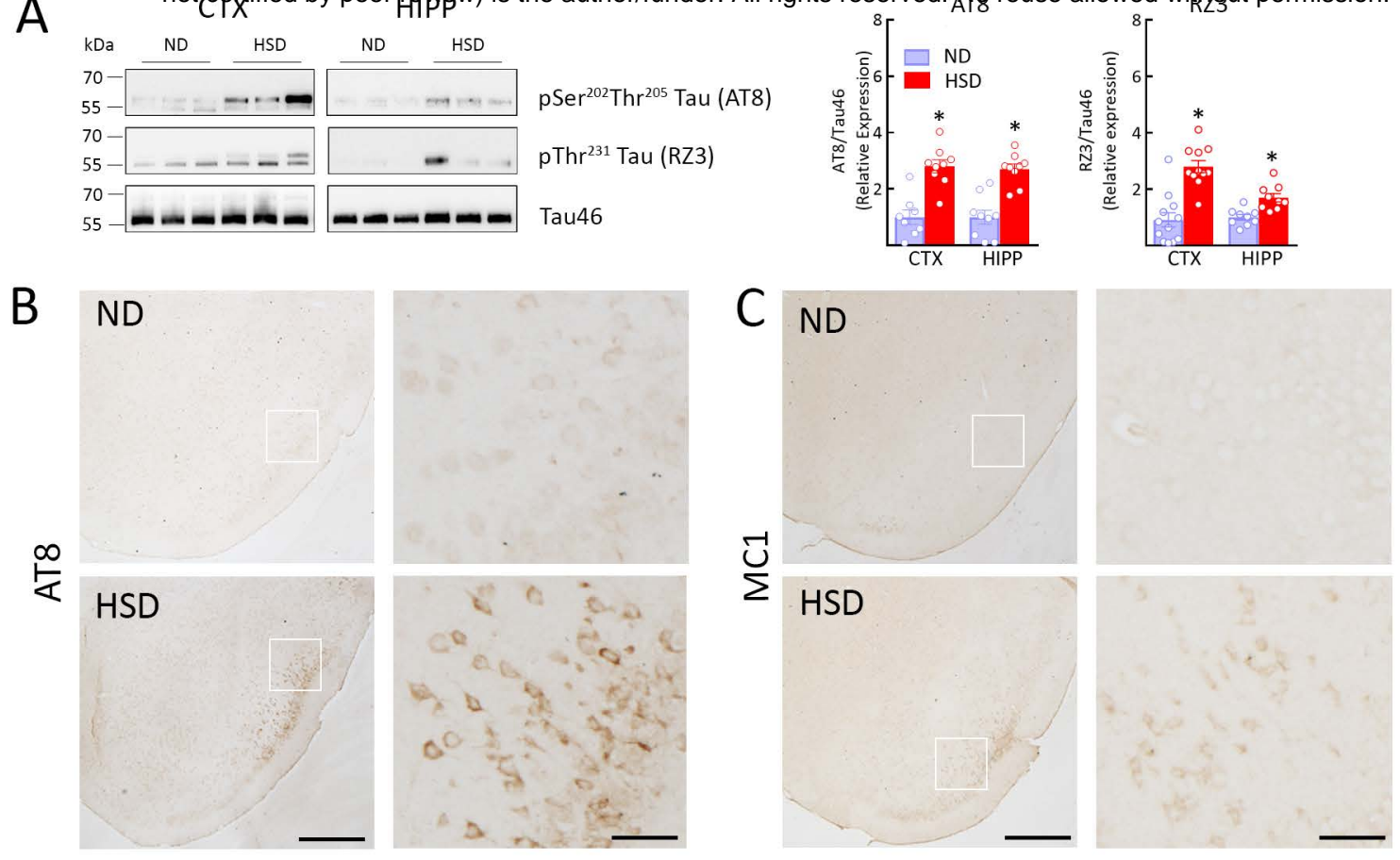

D
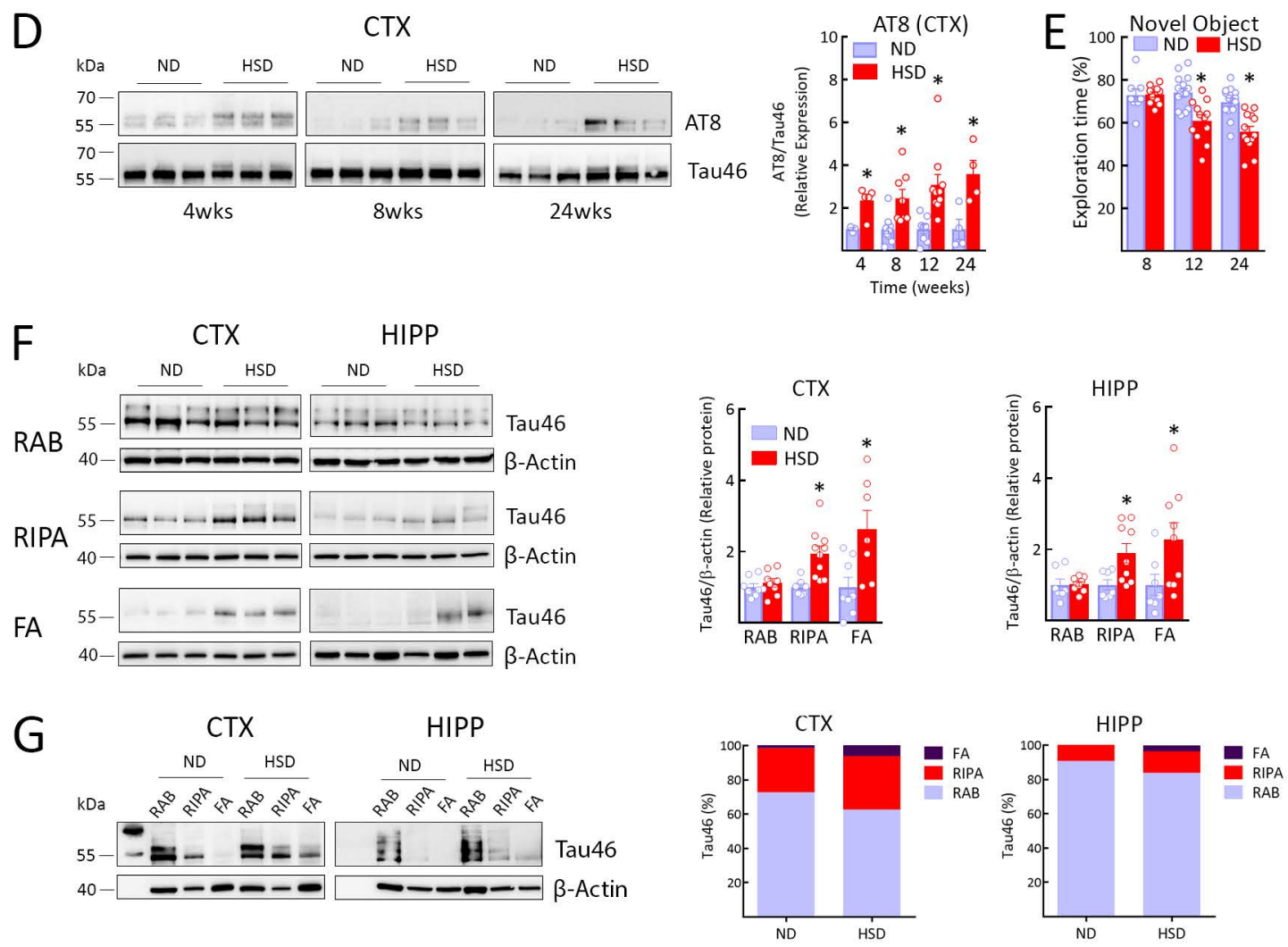

\section{Figure 1}

HSD increases tau phosphorylation and insoluble tau. A-B: HSD ( $\mathrm{NaCl} 8 \%$ ) increases tau phosphorylation on Ser $202 \mathrm{Thr}{ }^{205}$ (AT8) and $\mathrm{Thr}^{231}$ (RZ3) both in neocortex and hippocampus. (CTX: AT8, ND/HSD $n=8 / 9,{ }^{*} p=0.0003$ vs ND; RZ3, ND/HSD $n=12 / 11,{ }^{*} p<0.0001$ vs ND; HIPP: AT8, ND/HSD $n=9 / 9,{ }^{*} p=0.0005$ vs ND; RZ3, ND/HSD $n=9 / 9$, ${ }^{*} p=0.0008$ vs ND, two-tailed unpaired t-test). C: HSD increases AT8 and MC1 immunoreactivity in neuronal cell bodies of the piriform cortex (size bar=500 $\mu m$; $100 \mu m$ in inset). D: Time course of the increase in AT8 induced by HSD in the neocortex. The increase is observed at 4 weeks and is greatest at 24 weeks of HSD (AT8, 4 weeks: $\mathrm{ND} / \mathrm{HSD} n=3 / 5,{ }^{*} p=0.0357$ vs ND 4wks; 8 weeks: ND/HSD $n=9 / 8,{ }^{*} p=0.0016$ vs ND $8 w k s ; 24$ weeks: ND/HSD $n=4 / 4,{ }^{*} p=0.0286$ vs ND 24wks, two-tailed unpaired t-test). E: HSD induces deficits in recognition memory assessed by the novel object test, first observed at 12 weeks (Diet: ${ }^{*} p<0.0001$, Time: ${ }^{*} p=0.0002 ; 8$ weeks: ND/HSD $n=8 / 11 ; 12$ weeks: ND/HSD $n=16 / 12 ; 24$ weeks: ND/HSD $n=14 / 13$ mice/group, two-way ANOVA and Tukey's test). F: Extraction of total tau in RAB, RIPA and 70\% FA buffer in neocortex and hippocampus. HSD increases levels of tau extracted in RIPA and FA after 12 weeks of treatment indicating increased insolubility (CTX: RIPA, ND/HSD $n=7 / 10,{ }^{*} p=0.0032$ vs ND RIPA; FA, ND/HSD $n=8 / 7,{ }^{*} p=0.0146$ vs ND FA; HIPP: RIPA, ND/HSD $n=7 / 9,{ }^{*} p=0.0186$ vs ND RIPA; FA, ND/HSD $n=7 / 9,{ }^{*} p=0.0494$ vs ND FA, two-tailed unpaired t-test). G: Fractionation of total tau in RAB, RIPA, or $70 \%$ FA. HSD shifts tau from the more soluble RAB fraction to the less soluble RIPA and FA fractions (CTX: ND/HSD n=9/8, RAB, p=0.4234 vs ND, RIPA, $p=0.5414$ vs ND, FA, ${ }^{*} p<0.0325$ vs ND; HIPP: ND/HSD $n=5 / 6, R A B, p=0.2468$ vs ND, RIPA, $p=0.3290$ vs ND, FA, ${ }^{*}<<0.0152$ vs ND, two-tailed unpaired t-test). Immunoblots in $\mathbf{A}, \mathbf{D}, \mathbf{F}$ and $\mathbf{G}$ are cropped. Full gel pictures for immunoblots are shown in Extended Data Fig.5 and 6. Data are expressed as mean \pm SEM. 

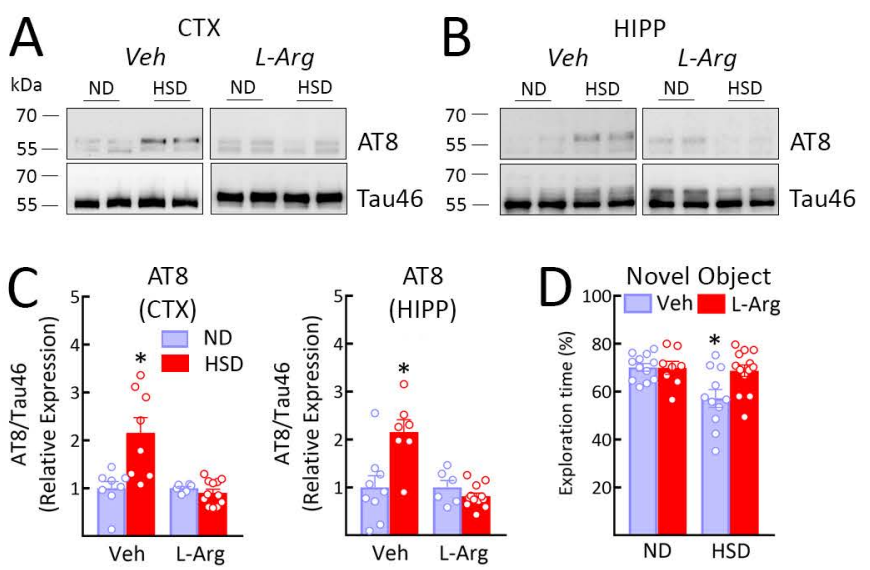

Figure 2

The NO precursor L-arginine prevents the increase in $\mathrm{p}$-tau induced by HSD. A-C: Administration of L-arginine $(10 \mathrm{~g} / \mathrm{L}$ in drinking water), starting at week 8 of $\mathrm{HSD}$ and continued through week 12 , suppresses AT8 accumulation in both neocortex and hippocampus (CTX: Vehicle, ND/HSD $n=8 / 8$, Diet: ${ }^{*} p=0.0060$, Treatment: ${ }^{*} p=0.0015$; HIPP: Vehicle, ND/HSD $n=9 / 7$, Diet: ${ }^{*} p=0.0145$, Treatment: ${ }^{*} p=0.0011$, two-way ANOVA and Bonferroni's test). D: L-arginine treatment improves the cognitive deficits induced by HSD (Veh - ND/HSD $n=12 / 10$, L-Arg - ND/HSD $n=6 / 11$; Diet: ${ }^{*} p=0.0156$, Treatment: ${ }^{*} p=0.0406$, two-way ANOVA and Tukey's test). Immunoblots in $\mathbf{A}$ and $\mathbf{B}$ are cropped. Full gel pictures for immunoblots are shown in Extended Data Fig.7. Data are expressed as mean \pm SEM. 

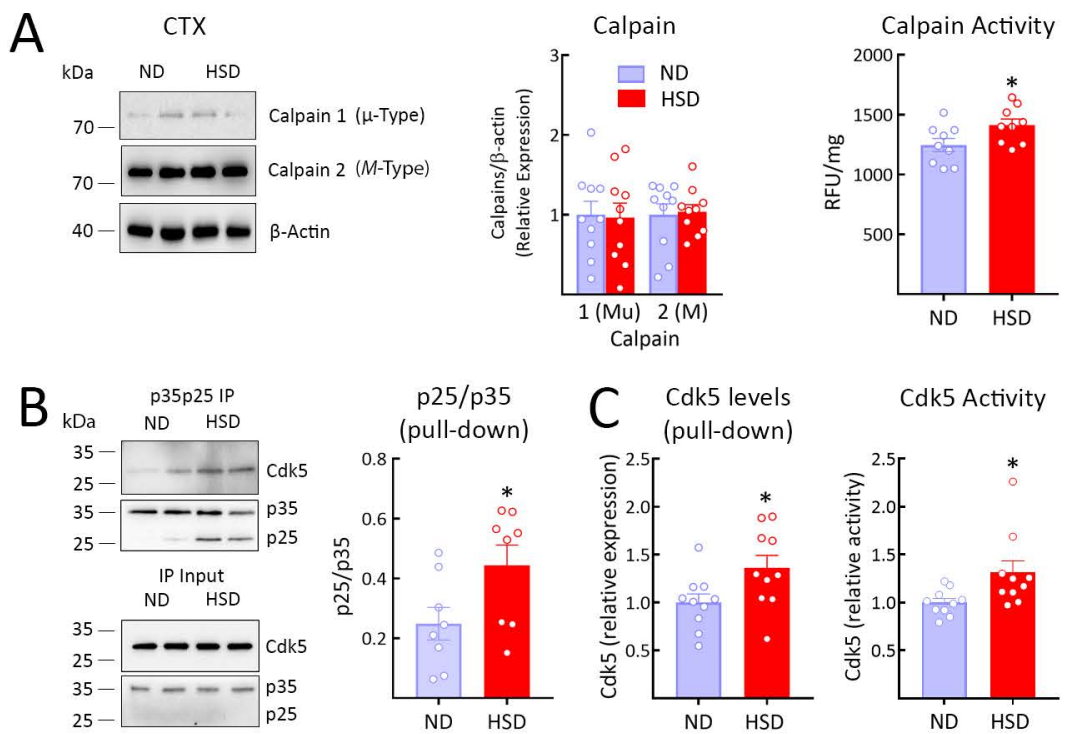

D
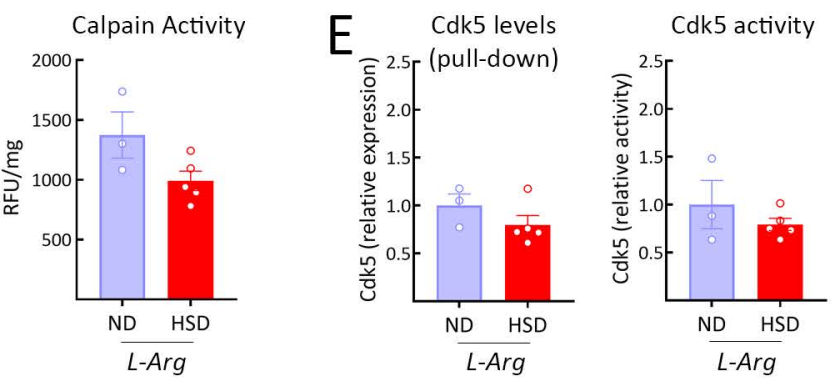

$\mathrm{F}$
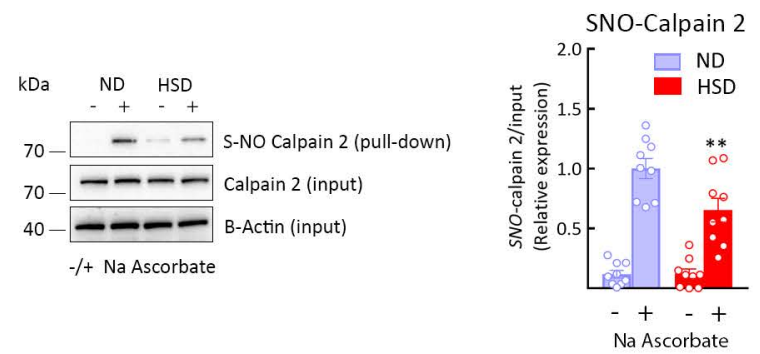

Figure 3

HSD induces activation of calpain and Cdk5, an effect associated with calpain denitrosylation. A: HSD did not alter calpain 1 or 2 expression (ND/HSD, $n=10$ ), but increased enzyme activity (ND/HSD $n=9 / 9,{ }^{*} p=0.0314$ vs ND, two-tailed unpaired t-test). B: Consistent with calpain activation, HSD increases the cleavage of p35 into $\mathrm{p} 25$ (ND/HSD $n=8 / 8,{ }^{*} \mathrm{p}=0.0379$ vs ND, two-tailed unpaired t-test). C: HSD increases the levels of Cdk5 bound to p35p25 (ND/HSD $n=10 / 10,{ }^{*} p=0.0232$ vs ND, two-tailed unpaired t-test) and Cdk5 activity (ND/HSD $n=10 / 10,{ }^{*} p=0.0115$ vs ND, two-tailed unpaired t-test). D-E: L-arginine administration counteracts the increase in calpain and Cdk5 activity induced by HSD (ND/HSD n=3/5). F: Nitrosylation of calpain 2, the predominant brain isoform of the enzyme (see A), is reduced in HSD mice (ND/HSD $n=9 / 9$, Diet: ${ }^{*} p=0.0189$; Treatment: ${ }^{*} p<0.0001$, two-way ANOVA and Tukey's test). Nitrosylation was assessed by the biotin switch assay. Immunoblots in $\mathbf{A}, \mathbf{B}$ and $\mathbf{F}$ are cropped. Full gel pictures for immunoblots are shown in Extended Data Fig.8. Data are expressed as mean \pm SEM. 
bioRxiv preprint doi: https://doi.org/10.1101/470666; this version posted November 14, 2018. The copyright holder for this preprint (which was
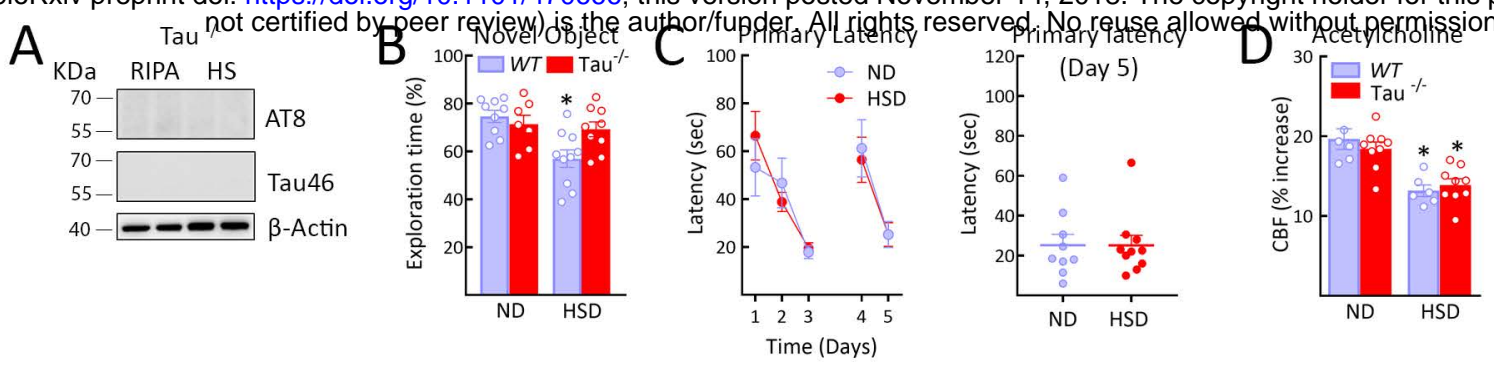

\section{$\mathrm{E}$}
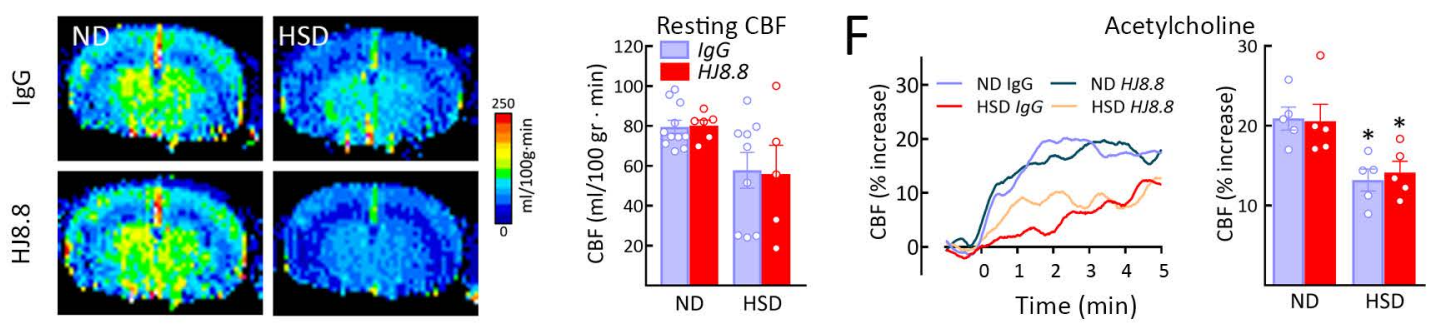

\section{G}
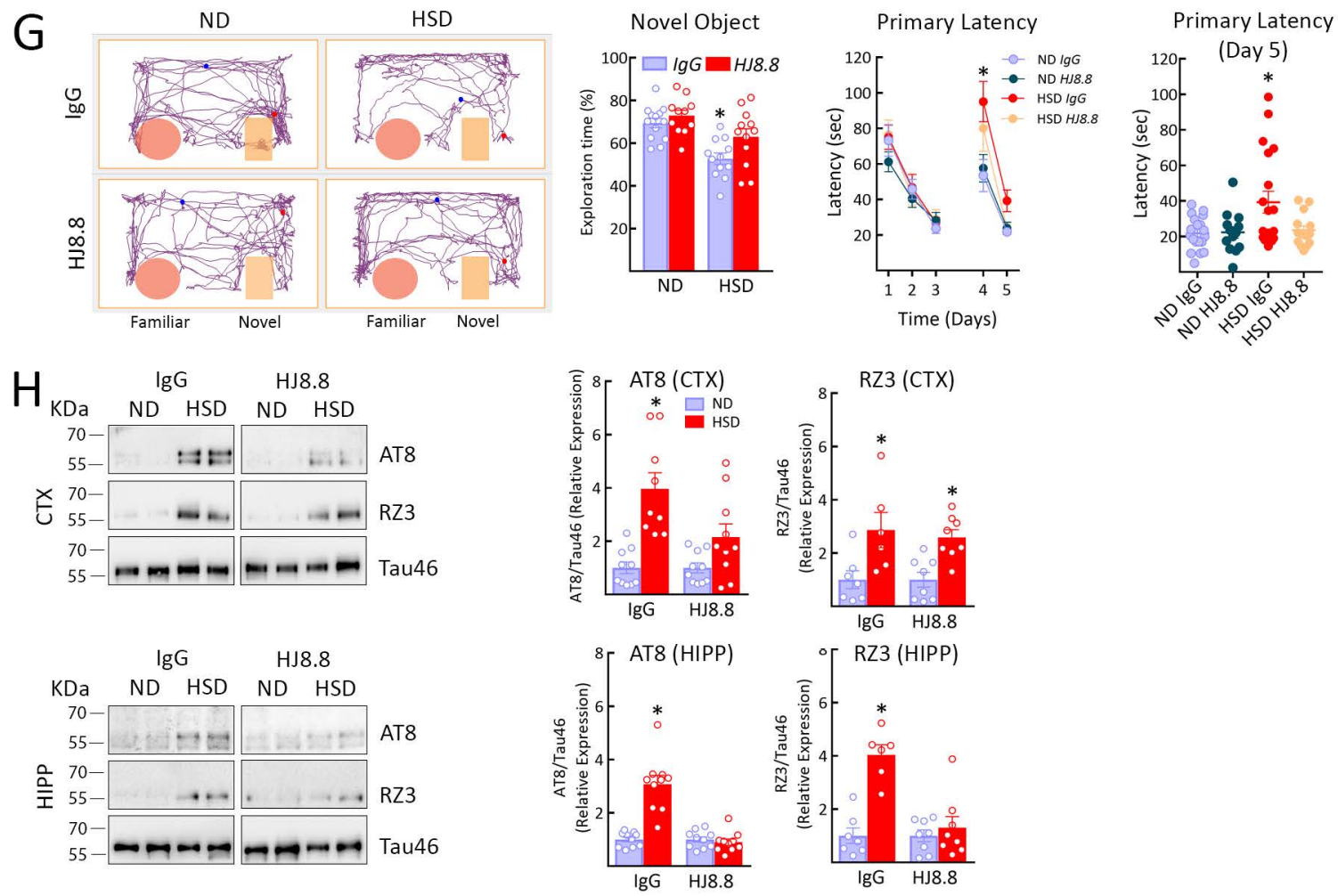

Figure 4

HSD-induced cognitive dysfunction is not observed in tau- mice and prevented by tau antibodies despite cerebrovascular insufficiency. A: AT8 and Tau46 are absent in tau- mice in both the RIPA and heat-stable RIPA fractions. B-C: HSD does not alter cognitive function in tau- mice, assessed by the novel object recognition test (WT: ND/HSD n=9/10; Tau-/-: ND/HSD n=7/9; Diet: ${ }^{*} p=0.0055$, Genotype: $p=0.1827$, two-way ANOVA and Tukey's test) or the Barnes maze (ND/HSD $n=9 / 10$, Diet: $p=0.9348$, Time: ${ }^{*} p<0001$, Two-way ANOVA and Tukey's test). D: Despite improved cognition, the CBF increase produced by neocortical application of acetylcholine in anesthetized mice equipped with a cranial widow, a response mediated by eNOS-derived NO, is still reduced in tau ${ }^{-/}$ mice (ND/HSD WT, $n=6 / 6$, Tau-/-, n=9/9; Diet: * $p<0.0001$, Genotype: $p=0.7920$, Two-way ANOVA and Tukey's test). CBF was measured by laser-Doppler flowmetry. E: Systemic administration of anti-tau antibodies (HJ8.8, 50mg/Kg/week i.p.) does not rescue the reduction in resting CBF induced by HSD, assessed by ASL-MRI (IgG: ND/HSD n=11/9; HJ8.8: ND/HSD n=6/5; Diet: * $p=0.0061$, Treatment: $p=0.9367$, two-way ANOVA and Tukey's test). F: Similarly, HJ8.8 administration does not prevent the attenuation of the CBF response to ACh induced by HSD (IgG: ND/HSD n=5/5; HJ8.8: ND/HSD n=5/5; Diet: * $p=0.0005$, Treatment: $p=0.8516$, two-way ANOVA and Tukey's test). G: HJ8.8 administration ameliorates the cognitive dysfunction induced by HSD both at the novel object test (IgG: ND/HSD n=13/12; HJ8.8: ND/HSD n=11/12; Diet: * $p<0.0001$, Treatment: * $p=0.0231$, two-way ANOVA and Tukey's test) and the Barnes maze (Primary Latency - IgG: ND/HSD n=19/19; HJ8.8: ND/HSD n=13/14; Time: * $p<0.0061$, Diet: * $p=0.0317$, repeated-measures two-way ANOVA and Tukey's test; Primary Latency - Day 5 - IgG: ND/HSD n=19/19; HJ8.8: ND/HSD n=13/14; Diet: *p=0.0320, Treatment: $p=0.0756$, two-way ANOVA and Tukey's test). H: HJ8.8 administration reduces AT8 levels in both neocortex and hippocampus (AT8, CTX - IgG: ND/HSD $n=10 / 9$; HJ8.8: ND/HSD $n=10 / 10$; Diet: * $p<0.0001$, Treatment: * $p=0.0313$; AT8 - HIPP - IgG: ND/HSD $n=10 / 10$; HJ8.8: ND/HSD $n=10 / 10$; Diet: * $p<0.0001$, Treatment: * $p<0.0001$, two-way ANOVA and Bonferroni's test) and RZ3 levels in the hippocampus but not neocortex (RZ3, CTX - IgG: ND/HSD n=7/7; HJ8.8: ND/HSD n=8/8; Diet: * $p=0.0005$, Treatment: $p=0.9586$, RZ3 - HIPP - IgG: ND/HSD $n=7 / 6$; HJ8.8: ND/HSD n=8/8; Diet: * $p<0.0001$, Treatment: * $p=0.0004$, two-way ANOVA and Bonferroni's test). Immunoblots in $\mathbf{A}$ and $\mathbf{H}$ are cropped. Full gel pictures for immunoblots are shown in Extended Data Fig.9. Data are expressed as mean \pm SEM. 